\title{
A Turkish Family with Loeys-dietz Syndrome and a Report of a Homozygous Patient with SMAD3 Pathogenic Variation
}

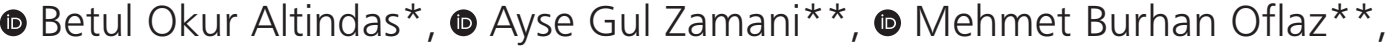 \\ ๑ ( Muhammed Gunes**, (1) Mahmut Selman Yildirim* \\ ${ }^{*}$ Necmettin Erbakan University, Department of Medical Genetics, Konya, Turkey \\ **Necmettin Erbakan University, Department of Pediatrics, Division of Pediatric Cardiology, Konya, Turkey
}

\section{Abstract}

Loeys-Dietz syndrome (LDS) is a rare autosomal dominant connective tissue disorder with multisystemic involvement caused by pathogenic genetic variations in the transforming growth factor- $\beta$ pathway. Here, we report a homozygous case with LDS. A newborn male patient who had congenital diaphragmatic hernia, aortic dilatation and talipes equinovarus was referred to our medical genetics polyclinic. After clinical evaluation, next generation sequencing analysis showed a homozygous C.859C>T pathogenic missense variation [R287W (p.Arg287Trp)] in the SMAD3 gene. It was confirmed that the parents harbor the variant heterozygously. Due to the autosomal dominant inheritance pattern, rarely seen biallelic individuals are expected to have severe clinical conditions. Since there was only one previous report of an individual harboring a homozygous SMAD3 variant in the literature; this case was presented to further enhance our understanding of LDS.

Keywords: Loeys-Dietz syndrome, LDS, SMAD3, TGF-beta

*Content of this report has been presented before in "14. Ulusal Tıbbi Genetik Kongresi Uluslararası Katılımlı" in November 20-22, 2020, online.

\section{Introduction}

Loeys-Dietz syndrome (LDS) is an autosomal dominant connective tissue disorder that is characterized by cardiovascular, skeletal, and craniofacial features as well as cutaneous findings. Significant clinical variability exists among the patients, even within the family members. TGFBR1, TGFBR2, TGFB2, TGFB3, SMAD2, $S M A D 3$ genes have been identified as the LDS-causing genes $(1,2)$. Since there is no specific clinical diagnostic criteria, the diagnosis is confirmed by molecular testing. While type 1 and 2 LDS are the most common and aggressive types, SMAD3 pathogenic variations are seen in 5 to $10 \%$ of patients, resulting in LDS type 3 (3). As an autosomal dominant disease, biallelic patients are uncommon and expected to have a severe phenotype. In this case, the goal was to improve our understanding of LDS by reporting a homozygous case and addressing potential differences in severity.

\section{Case Report}

A two-day-old male patient was referred to the Medical Genetics Department with club foot deformity, floppiness and congenital diaphragmatic hernia (October, 2019). He was born prematurely at 35 weeks of gestation. Physical examination showed; he had microretrognathia, low-set ears, medial deviation of the wrists, bilateral inguinal hernia, genu varum deformity and bilateral talipes equinovarus (Figure 1). In the echocardiography of the patient, a $3.2 \mathrm{~mm}$ atrial septal defect with left-to-right shunt, a $3 \mathrm{~mm}$ patent ductus arteriosus, dilatation of the ascending aorta (Z score: +4.7 ), tortuosity and marked elongation of the descending aorta after the isthmus were

Address for Correspondence: Betul Okur Altindas 

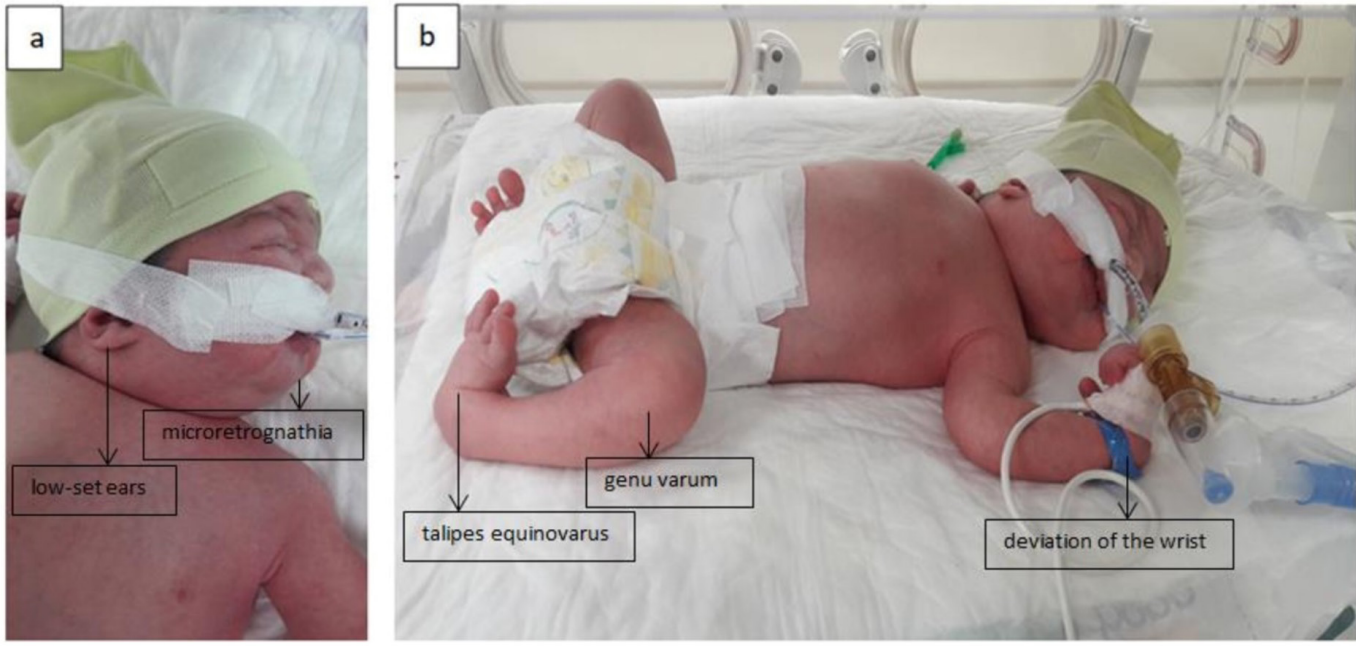

Figure 1. The proband's photographs show: a) microretrognathia, low-set ears; and b) skeletal findings, including a medial deviation of the left wrist, severe genu varum deformity, and bilateral talipes equinovarus.

observed. The interatrial septum was also aneurysmatic (Figure 2). The parents, who were 24 and 28 years old, had no complaints about their health and were consanguineous, being first-degree cousins. The first pregnancy in the family resulted in a missed abortus within the first trimester. The second child was born with severe anomalies, including club foot, complete atrioventricular canal defect and an absent pulmonary valve with pulmonary stenosis. She died on the fifth day of her life, without any genetic testing or sample collection. The third child only had mild joint laxity and intermittent eczema and the fourth one was our proband. The patient had undergone an operation for his diaphragmatic hernia, but he died due to cardiac complications on the eleventh day. A peripheral blood sample was taken and his karyotype analysis resulted (as) normal. Targeted next-generation sequence analysis using the NEXTflex Aortopathy panel kit (PerkinElmer Inc.) for ACTA2, COL3A1, FBN1, SMAD3, TGFBR2, SLC2A10, TGFB2, MYH11, CBS, SMAD2, FBN2, TGFBR1 genes was performed on the Illumina MiniSeq platform. The analysis showed a homozygous C.859C $>T$ missense variation (R287W [p.Arg287Trp]) in the SMAD3 gene (RefSeq: NM_005902.4; Transcript ID: ENST00000327367.4). This variant was confirmed by Sanger sequencing analysis (Figure 3). The parents' molecular testing revealed that both of them harbor the same variation heterozygously. The father had typical manifestations of LDS; including turricephaly, hypertelorism, low-set ears, prognathism, bifid uvula, arachnodactyly, joint laxity, pectus carinatum, tall stature (198 cm of measured height) and dilatation
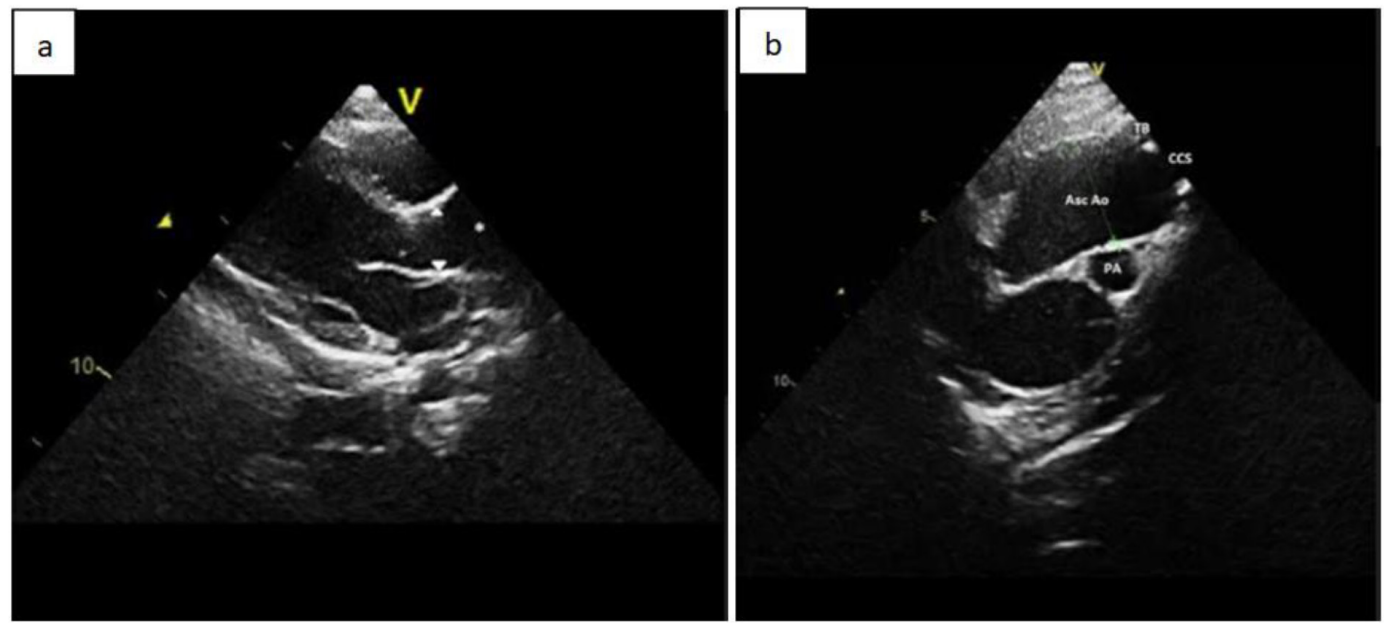

Figure 2. a) The proband's echocardiographic image of the parasternal long section shows the ascending aorta $\left({ }^{*}\right)$ is significantly wider than the aortic root (arrowheads). b) An echocardiographic image of the suprasternal section shows that the pulmonary artery is normal in width, but the ascending aorta (Asc Ao) is significantly dilated and wide.

TB: Truncus brachiocephalicus, CCS: Carotis communis sinistra 


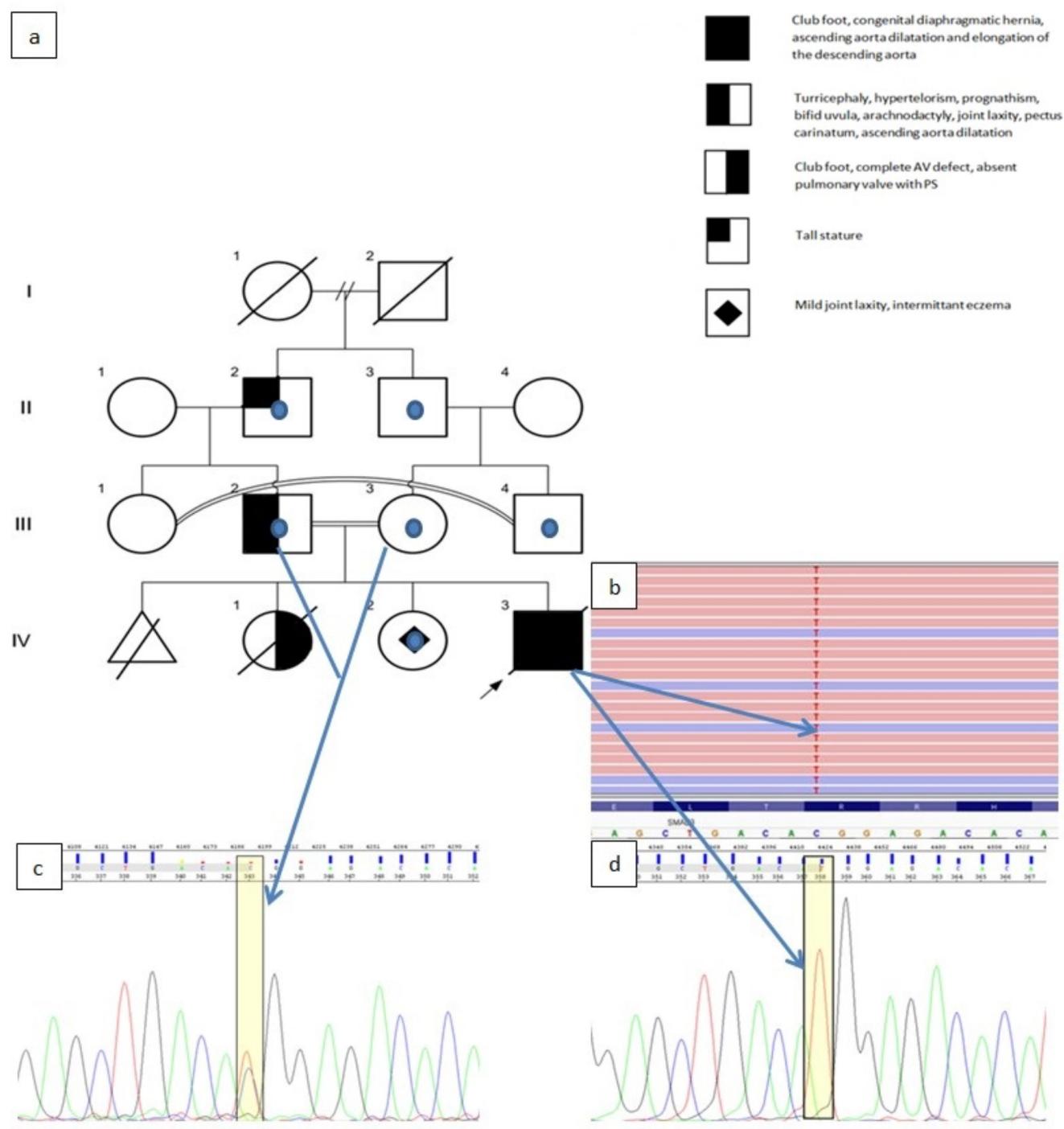

Figure 3. The pedigree of the family (individuals with a blue dot were shown to harbor the SMAD3 variation.) and their DNA sequencing results.

\section{a) The individual properties of the family members are as follows:}

II-2: A 47-year-old male with a tall stature (measured height is $198 \mathrm{~cm}$ ), who was found to have a heterozygous SMAD3 R287W variation.

II-3, III-3, III-4: Individuals who were found to be heterozygous for the SMAD3 R287W variant had no LDS-related symptoms or findings so far. Their echocardiography was normal.

III-2: A 31-year-old patient who had craniofacial and skeletal findings of Loeys-Dietz syndrome, including tall stature (measured height was $198 \mathrm{~cm}$ ), turricephaly, hypertelorism, low-set ears, prognathism, bifid uvula, arachnodactyly, joint laxity, pectus carinatum, and ascending aorta dilatation.

IV-1: Newborn with club foot, complete atrioventricular defect, absent pulmonary valve with pulmonary stenosis; died in the first week of life without having a genetic test.

IV-2: A 5-year-old female with normal echocardiography, who had mild joint laxity and eczematoid dermatitis. A heterozygous SMAD3 R287W variation was detected.

IV-3 (proband): newborn with club foot, congenital diaphragmatic hernia, ascending aorta dilatation (Z score: $+4,7)$, and elongation of the descending aorta. A homozygous SMAD3 R287W variation was observed.

b) The proband's raw IGV analysis data. All reads show a homozygous change from the reference allel (C) to the variant allel (T), which is visible in all reads (reads are depicted as lines, with red indicating the forward strand and blue indicating the reverse strand). c) The father's Sanger sequencing plot for the chr15:67473779 region, with several base pairs surrounding it. Each peak represents a nucleotide read (red peak: T, blue peak: C, black peak: G, green peak: A). A highlighted region depicting the heterozygous change was observed.

d) Proband's Sanger sequencing plot for the same region In the highlighted region, a homozygous change was observed.

AV: atrioventricular, LDS: Loeys-Dietz syndrome, PS: Pulmonary stenosis, IGV: Integrative genomics viewer 

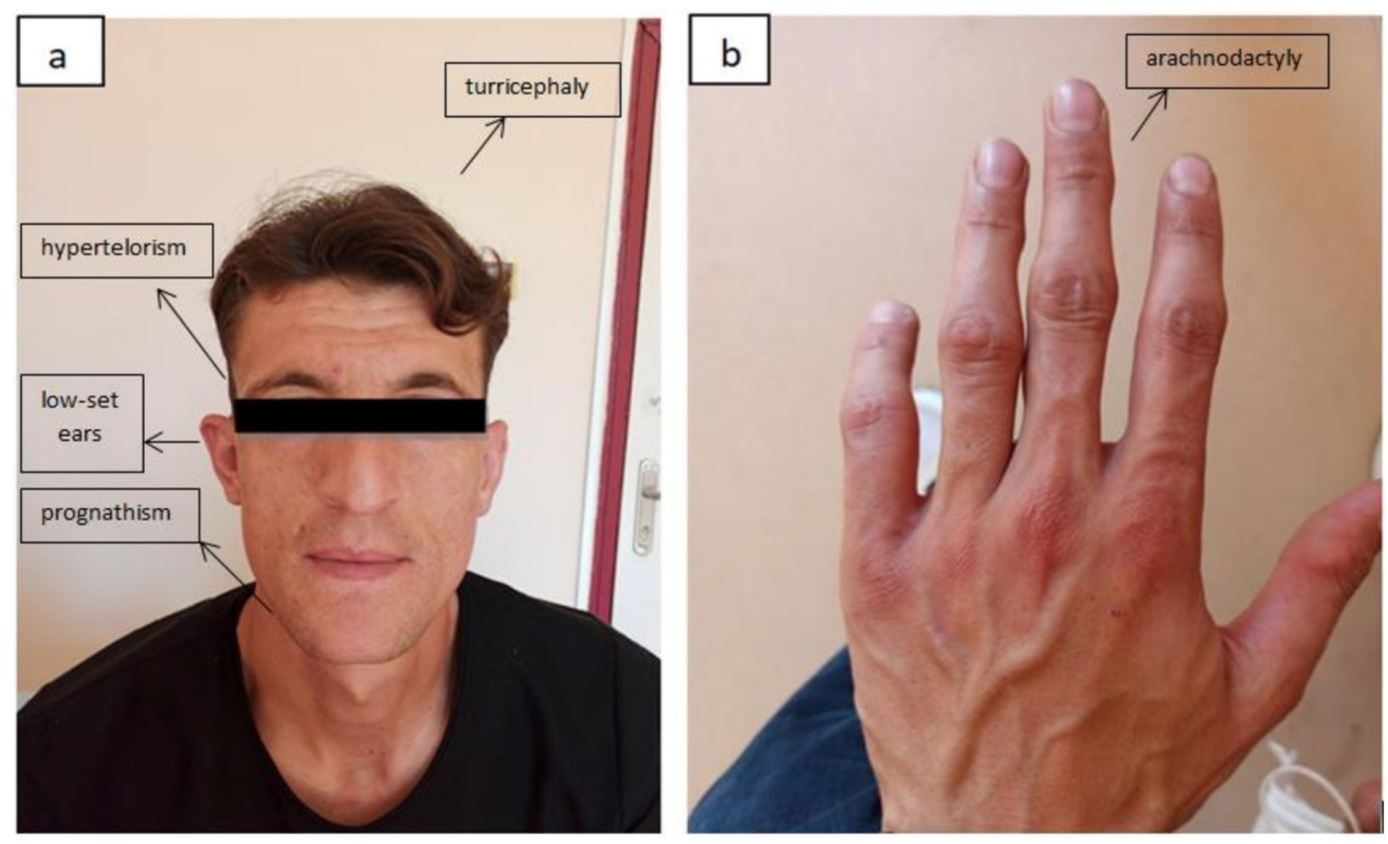

Figure 4. Photographs of the proband's father, a) showing turricephaly, hypertelorism, low-set ears, and a prominant chin; b) arachnodactyly of his left hand. (Informed consent regarding publication of the photos was taken from the patient)

of the ascending aorta up to $4.5 \mathrm{~cm}$ (Figure 4). Unlike the father, the mother, who had no symptoms or findings, was considered non-penetrant. The five-year-old sister of the proband who also had the heterozygous diseasecausing variant had no known medical history. She had mild joint laxity and intermittent eczema on the anterior surface of her arms; her echocardiography was normal. Pedigree analysis demonstrated that the grandfathers of both sides of the patient were brothers. The brother of the proband's mother and the sister of the proband's father were also married. Since they were candidates for having this variant, Sanger sequencing analysis was performed on them all. It was confirmed that the grandfathers on both sides and the maternal uncle of the patient had the same variation. Clinical examination showed only the paternal grandfather, whose echocardiography was normal, had a tall stature (198 cm of measured height), but other family members had no complaints or findings. Further vascular testing and close cardiologic monitoring were advised. Appropriate informed consent for the publication of the patient's and their data was taken from the parents.

\section{Discussion}

LDS is a rare connective tissue disorder characterized by vascular tortuosity and multisystemic involvement. All previously reported variants were found to be essential for the TGF- $\beta$ signalling pathway. Our patient had a pathogenic variant in the SMAD3 gene encoding a protein named exactly after its gene, SMAD3. It functions as an effector protein, transmitting chemical signals from the cell surface to the nucleus and acting as a direct mediator of TGF- $\beta$ receptor mediated transcriptional activation (4). The variation in our patient resulted in a shift from arginine to triptophan in the primary structure of the relevant protein. Because the arginine residue is well-conserved across species and there is a moderate physicochemical difference between these two amino acids, in silico analysis predicted this variation would be damaging to the protein structure and/or function. This variant was not found in the population databases and was previously reported as "pathogenic" in the ClinVar database (5). As a consequence, it was interpreted as pathogenic.

Approximately $25 \%$ of the probands were shown to have an affected parent. Therefore, a clinical examination and molecular testing of the parents are needed. Our homozygous patient had both parents as carriers. By definition, an allele determining the phenotype of a heterozygote individual is dominant. Homozygotes for dominant phenotypes are not often seen in medical practice because matings that could produce homozygous offspring are very rare (6). In this family, both our patient and his sister, who had severe skeletal and cardiac conditions, could not survive the newborn period. They seem to represent a very severe form of the syndrome, indicating they probably had the same genotype. To our knowledge, there has only been one previous report of a proband with a biallelic SMAD3 variant (7). That case reported by Baskin et al. (7) had notable musculoskeletal complications, including repetitive bone fractures, kyphoscoliosis, severe pectus excavatum, 
and dysmorphic features concordant with the syndrome. The variant detected was intronic and predicted to affect splicing, while our case had a missense variant. In a study, Chesneau et al. (8) compared truncating and missense variations of the SMAD3 gene and found they both cause aortic disease with no statistically significant difference. However, considering that the homozygote patient was in his teenage years and our case died in the second week of his life, homozygous cases could also have highly variable clinical courses, in terms of expression of the SMAD3 variants. In the literature, Camerota et al. (9) characterized LDS genotypic variants and showed more than half of the patients harboring SMAD3 variations had hernia, pectus deformity, malar hypoplasia, mitral valve defects, and aortic root aneurysms, while none of them had strabismus or cervical malformation. Only the father, who had facial dysmorphism, skeletal findings, and aortic dilatation, and the grandfather, who was tall, had LDS-associated clinical presentation among our heterozygote cases. None of our patients, regardless of age, had osteoarthritis, indicating that the SMAD3 gene has incomplete penetrance and variable expression (10).

LDS is a rare cause of aortic aneurysm; however, genetic testing is needed if the patient has accompanying skeletal and/or skin findings. Phenotypic variability in this disease has emerged as a fact, as more patients are identified. Because SMAD3 gene mutations are rare causes of LDS and the majority of patients are heterozygous, reports of rare biallelic individuals are considered significant. It is clearly beneficial to keep homozygosity in mind, to facilitate early and accurate diagnosis of a newborn with a severe condition, especially in the case of consanguinity.

\section{Ethics}

Informed Consent: Informed consent regarding publication of the photos was taken from the family.

\section{Authorship Contributions}

Concept: B.O.A., A.G.Z., Design: M.B.O., B.O.A., Data Collection or Processing: M.G., B.O.A., Analysis or Interpretation: B.O.A., A.G.Z., Literature Research: M.S.Y., B.O.A., Writing: B.O.A.
Conflict of Interest: No conflict of interest was declared by the authors.

Financial Disclosure: The authors declared that this study received no financial support.

\section{References}

1. Loeys BL, Chen J, Neptune ER, et al. A syndrome of altered cardiovascular, craniofacial, neurocognitive and skeletal development caused by mutations in TGFBR1 or TGFBR2. Nat Genet 2005;37:275-81.

2. https://www.omim.org/phenotypicSeries/?phenotypicSer iesNumber=PS607086,PS609192.(accessed at 01.12.2021)

3. Loeys BL, Dietz HC. Loeys-Dietz Syndrome. In: Adam MP, Ardinger $\mathrm{HH}$, Pagon RA, et al. editors. GeneReviews $\AA$. Seattle (WA): University of Washington, Seattle; 2008.

4. Massagué J. TGF $\beta$ signalling in context. Nat Rev Mol Cell Biol 2012;13:616-30.

5. https://www.ncbi.nlm.nih.gov/clinvar/variation/30306/ (accessed at 01.12.2021)

6. Nussbaum RL, Mclnnes RR, Willard HF. Thompson \& Thompson Genetics in Medicine. 8th ed. Philadelphia: Elsevier; 2016. p. 114.

7. Baskin SM, Morris SA, Vara A, Hecht JT, Farach LS. The first reported case of Loeys-Dietz syndrome in a patient with biallelic SMAD3 variants. Am J Med Genet A 2020;182:2755-60.

8. Chesneau B, Edouard T, Dulac Y, et al. Clinical and genetic data of 22 new patients with SMAD3 pathogenic variants and review of the literature. Mol Genet Genomic Med 2020;8:e1132.

9. Camerota L, Ritelli M, Wischmeijer A, et al. Genotypic Categorization of Loeys-Dietz Syndrome Based on 24 Novel Families and Literature Data. Genes (Basel) 2019;10:764.

10. van de Laar IM, Oldenburg RA, Pals G, et al. Mutations in SMAD3 cause a syndromic form of aortic aneurysms and dissections with early-onset osteoarthritis. Nat Genet 2011;43:121-6. 